\title{
Radiotherapy for small cell carcinoma of the esophagus: outcomes and prognostic factors from a retrospective study
}

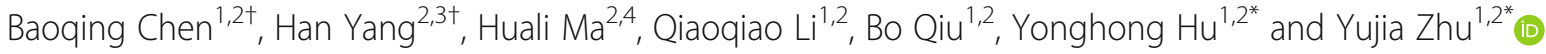

\begin{abstract}
Background: Small cell carcinoma of the esophagus (SCCE) is characterized by its progressive feature and poor prognosis. There is no consensus on a standard therapeutic modality for SCCE. In this study, we aimed to characterize the outcomes of primary SCCE patients treated by radiation therapy as part of treatment and investigate prognostic factors.
\end{abstract}

Methods: We retrospectively analyzed the data of 42 SCCE patients who were treated by RT as part of treatment at the Sun Yat-sen University Cancer Center from 2001 to 2014. The Kaplan-Meier and log-rank method were used to analyze survival. Cox's hazard regression model was applied to determine prognostic factors.

Results: Of the 42 enrolled patients, 25 had limited disease (LD) and 17 with extensive disease (ED). The overall response rate $(C R+P R)$ was $60.0 \%(21 / 35)$. The median overall survival time (OS) for whole and LD group were 12.9 and 36.8 months. The 1-, 3- and 5-year OS rates of the whole cohort were 64.9, 31.3, and 13.9\%, respectively. OS was significantly longer in patients with ECOG performance score (ECOG PS) $<2(p=0.001)$, lesion length $\leq 5 \mathrm{~cm}$ $(p=0.001)$, and LD $(p=0.049)$. In the patients with LD, multivariate analysis indicated that combined with chemotherapy $(P=0.046)$ and higher radiation dose $(P=0.027)$ predicted better prognosis in OS. The overall rate of grade 3-4 toxicities in the whole cohort was 37.5\%. In total, 65\% (17/26) patients with recurrent disease died with the metastasis with or without the primary recurrence.

Conclusion: RT was one of the effective and safe treatments for locoregional control of SCCE. Lower ECOG PS score, shorter lesion length, treated with chemotherapy, and a higher dose of RT were identified as favorable independent prognostic factors.

Keywords: Small cell carcinoma of esophagus, Radiation therapy, Chemotherapy, IMRT, Prognosis

\section{Introduction}

Small-cell carcinoma is one kind of highly malignant neoplasm that usually originates from the lung. The first case of primary small cell carcinoma of the esophagus (SCCE) was reported by Mckeown in 1952 [1]. SCCE is characterized by its aggressive feature with poor prognosis and is distinct from the squamous cell carcinoma or adenocarcinoma of the esophagus, but similar to small

\footnotetext{
* Correspondence: huyh@sysucc.org.cn; zhuyuj@sysucc.org.cn ${ }^{\dagger}$ Baoqing Chen and Han Yang contributed equally to this work. ${ }^{1}$ Department of Radiation Oncology, State Key Laboratory of Oncology in South China, Collaborative Innovation Center of Cancer Medicine, Sun Yat-sen University Cancer Center, Guangzhou, 510060, P. R. China Full list of author information is available at the end of the article
}

cell carcinoma arising from lungs or other organs. A consensus on the standard therapeutic modality for SCCE has not been established, but based on retrospective studies, a multimodal therapeutic approach combining surgery, radiotherapy (RT), and chemotherapy is recommended $[2,3]$. When combined with chemotherapy, RT is considered a major local therapy approach for treating patients with localized or locoregional disease. Though RT is common in clinical practice in western countries, esophagectomy is more widely used compared to RT as the treatment for localized SCCE in China [2, 4]. A previous retrospective study of all the SCCE cases in our center from September 1990 to June 2011 showed that of 64 SCCE patients, only 15 (26.7\%) patients 
received RT [5]. Due to increasing evidence showing its non-inferiority to esophagectomy, RT technology was developed over the years in China for treating SCCE. In this retrospective study, we focused on SCCE cases who had received RT and explored their outcomes and prognostic factors.

\section{Patients and methods}

\section{Patients}

We retrospectively reviewed 9547 cases of esophagus carcinoma at Sun Yat-sen University Cancer Center from June 2001 to December 2014. Among these cases, $110(1.15 \%)$ patients were pathologically diagnosed as SCCE. Of these 110 patients, 45 (40.9\%) had received RT as part of treatment. The inclusion criteria were as follows: 1) age between 18 and 75 years; 2) Eastern Cooperative Oncology Group (ECOG) performance status (PS) score $\leq 3$; 3) received RT as part of the treatment; and 4) absence of previous thoracic RT. Finally, 42 patients with complete medical records were enrolled for analysis in this retrospective study.

Medical records retrieved were examined for medical history and physical examination; complete blood count and serum chemistry profile; electrocardiogram; bariumswallow examination; contrast-enhanced computed tomography (CT) scan of the neck, chest and upper abdomen, endoscopic ultrasound, ultrasound of the cervical lymph node, PET-CT, radioactive isotope bone scans, if available. The disease stage was presented as either a limited-stage disease (LD) or an extensive-stage disease (ED) according to the Veteran's Administration Lung Group's 2-stage classification scheme (VALSG) for primary pulmonary small cell lung cancer (SCLC) [6]. LD is defined as a tumor confined within a localized anatomical region, which can be safely encompassed within a radiation field and ED is defined as a tumor outside of the local regional region. Tumor location is defined based on the UICC 1987 standard.

The Ethics Committee of Sun Yat-sen University Cancer Center reviewed and approved this study, which was performed according to the principles of the Helsinki Declaration. Informed consent was obtained from each patient for the collection of clinical information at the first visit.

\section{Treatment}

For RT administration, a vacuum cradle for immobilization was made with the patient in a supine position. Patients were scanned from the first cervical vertebra (C1) to the third lumbar vertebra (L3) level. CT scan was performed with $5 \mathrm{~mm}$ thick slices. The gross tumor volume (GTV) consisted of tumor shown by CT scans or PET/CT or endoscopy. Lymph nodes were defined as positive nodes if it exhibited any of the following features on $\mathrm{CT}$ : short axis $\geq$
$10 \mathrm{~mm}$, distribution in a cluster of lymph nodes, infiltrative margin, or central necrosis. Lymph nodes that demonstrated high uptake on the PET/CT scan were also included in the GTV, regardless of size. The clinical target volume (CTV) comprised the original tumor or anastomosis site, supraclavicular, and station 1-5 and 7 lymph nodes. The plan target volume (PTV) 1 was defined as the GTV plus a margin of 5 $\mathrm{mm}$ and PTV2 was defined as the CTV plus a margin of 5 $\mathrm{mm}$ in all directions, respectively. Three-Dimensional Conformal Radiation Therapy (3D-CRT) treatment plans were calculated by Pinnacle planning system and Intensity Modulation Radiated Therapy (IMRT) treatment plans were calculated by the Monacle planning system. All patients were treated with a 6-MV linear accelerator. The prescribed dose was generally 50-66 Gy for PTV1 and 42-50 Gy for PTV2. Dose constraints for critical organs were spinal cord $<45$ Gy; mean lung dose $<17$ Gy and lung dose V $20<30 \%$.

\section{Statistics}

Tumor response was assessed using Response Evaluation Criteria in Solid Tumors 1.1 (RECIST 1.1). The Overall Survival (OS) time of patients was calculated from the start of initial treatment to the date of death or last follow-up and calculated and compared using the Kaplan-Meier method and the log-rank p test. Multivariate analysis was performed using Cox regression to analyze the prognostic factors. $P$ value $<0.05$ was considered significant. All statistical analysis was performed using SPSS 22.0 software.

\section{Results}

Patient demographic and baseline characteristics

Clinical data including patient demographics and tumor characteristics are outlined in Table 1 . The median age of patients was 55 years (range: 42-72 years) with 29 (69\%) male and $13(31 \%)$ female patients. Almost half (20/47.6\%) patients had a history of heavy smoking (smoking index $\geq 400$ ) or risky diets, which include heavy drinking, hot food or salt-preserved foods. Twenty-nine (69.0\%) patients had an Eastern Cooperative Oncology Group (ECOG) performance status (PS) of $0-1$, while $12(31.0 \%)$ patients had an ECOG PS of $2-3$. Middle thoracic SCCE was observed in 21(50.0\%) patients and two cases had multi-origins SCCE. Histological analysis showed 39 (92.9\%) patients with only small cell carcinoma whereas two patients had small cell carcinoma coexisting with squamous cell carcinoma, and another one with cardiac adenocarcinoma as well. At the time of diagnosis, 25 (59.5\%) had LD and the other 17 (40.5\%) patients had ED. Brain metastasis was not detected at the time of diagnosis but developed in two patients during follow-up. 
Table 1 Patient information and tumor characteristics

\begin{tabular}{|c|c|c|}
\hline & No. & Percentage $\%$ \\
\hline \multicolumn{3}{|l|}{ Sex } \\
\hline Male & 29 & 69.0 \\
\hline Female & 13 & 31.0 \\
\hline \multicolumn{3}{|l|}{ Age (55; range: $42-72$ years) } \\
\hline$<60$ years & 24 & 57.1 \\
\hline$\geq 60$ years & 18 & 42.9 \\
\hline \multicolumn{3}{|l|}{ Smoking history } \\
\hline yes & 20 & 47.6 \\
\hline no & 22 & 52.4 \\
\hline \multicolumn{3}{|l|}{ Risky diet } \\
\hline yes & 20 & 47.6 \\
\hline no & 22 & 52.4 \\
\hline \multicolumn{3}{|l|}{ ECOG PS } \\
\hline $0-1$ & 29 & 69.0 \\
\hline $2-3$ & 13 & 31.0 \\
\hline \multicolumn{3}{|l|}{ Location } \\
\hline cervical & 0 & 0 \\
\hline upper thoracic & 10 & 20.8 \\
\hline middle thoracic & 21 & 50.0 \\
\hline lower thoracic & 9 & 21.4 \\
\hline multiple & 2 & 4.8 \\
\hline \multicolumn{3}{|l|}{ Length (cm) } \\
\hline$\leq 5$ & 20 & 47.6 \\
\hline$>5$ & 22 & 52.4 \\
\hline \multicolumn{3}{|l|}{ Stage } \\
\hline extensive-disease & 17 & 40.5 \\
\hline limited-disease & 25 & 59.5 \\
\hline \multicolumn{3}{|l|}{ Pathology } \\
\hline Pure SCCE & 39 & 92.9 \\
\hline Coexisting with SCC & 2 & 4.8 \\
\hline Coexisting with Adenocarcinoma & 1 & 2.4 \\
\hline \multicolumn{3}{|l|}{ Radiation technology } \\
\hline 3DCRT & 24 & 57.1 \\
\hline IMRT & 18 & 42.9 \\
\hline \multicolumn{3}{|l|}{ Radiation Dose(58; Range: 42-66Gy) } \\
\hline$<56 \mathrm{~Gy}$ & 15 & 35.7 \\
\hline$\geq 56 \mathrm{~Gy}$ & 27 & 64.3 \\
\hline
\end{tabular}

Abbreviations: ECOG PS: the Eastern Cooperative Oncology Group Performance Status Scale, SCCE: Small Cell Carcinoma of esophagus, SCC:squamous cell carcinoma, 3DCRT: 3-Dimensional Conformal Radiation Therapy, IMRT: Intensity Modulation Radiated Therapy

\section{Treatment}

Of the 42 patients who received RT, 24 (57.1\%) received 3DCRT treatment, and the remaining 18 (42.9\%) patients received IMRT treatment. The median RT dose was 58 Gy that ranges from 42 to $66 \mathrm{~Gy}$. The treatment regimens for patients with LD or ED are listed in Table 2. In the 25 patients with $\mathrm{LD}$, seven patients received RT only, 13 patients were treated with RT combined with chemotherapy, while the left five received adjuvant RT \pm chemotherapy after surgery. Prophylactic brain radiation at a dose of $30 \mathrm{~Gy}$ in 10 fractions was performed in two patients with LD. Of the 17 patients with ED, eight patients received RT alone, five patients were treated with RT combined with chemotherapy, and the left four patients received RT with chemotherapy and surgery (two received adjuvant $\mathrm{RT}$ after esophagectomy, and two received pre-operation RT).

\section{Tumor response rate and survival}

Tumor response was assessed at two to 3 months after the completion of treatment. Among those 35 patients who are eligible for response evaluation (seven patients with adjuvant therapy after surgery were excluded), five patients achieved complete remission (CR) and 16 achieved partial remission (PR). The overall response rate $(\mathrm{CR}+\mathrm{PR})$ was $60.0 \%(21 / 35)$. Eight patients achieved stable disease (SD), and six achieved progressive disease (PD). The overall disease control rate is $83 \%(29 / 35)$. There was no evidence of relapse in seven patients who received adjuvant RT after esophagectomy.

The OS rates at 1-, 3- and 5-years in the whole cohort were $64.9,31.3$, and $13.9 \%$, respectively, with a median OS of 12.9 months (Fig. 1a). The OS rates at 1-, 3- and 5years in the subgroup of patients with LD were 78, 45.5, and $18.2 \%$, respectively, with a median OS of 36.8 months, whereas the 1-year and 3-years OS rates for ED patients decreased dramatically to 47.1 and $9.2 \%$, accompanied by decrease in median OS to 11.0 months (Fig. 1d).

At the time of last observation, 16 patients survived with or without the primary disease, while 26 patients died of tumor recurrence or metastases. In patients with LD, four patients died of local recurrence disease, and eight patients died of metastases disease. Similarly, in patients with ED, five patients died of local recurrence disease and nine patients died of metastases disease. In total, $65 \%(17 / 26)$ patients died with the metastasis with or without the primary recurrence.

Table 2 Treatment regimen of 42 SCCE patients

\begin{tabular}{llll}
\hline $\begin{array}{l}\text { Treatment } \\
\text { group }\end{array}$ & No. & ED & All(\%) \\
\cline { 2 - 4 } & LD & 8 & $15(35.7 \%)$ \\
\hline $\mathrm{R}$ & 7 & 5 & $18(42.9 \%)$ \\
$\mathrm{R}+\mathrm{C}$ & 13 & $4^{*}$ & $9(21.4 \%)$ \\
$\mathrm{S}+\mathrm{R} \pm \mathrm{C}$ & 5 & 17 & 42 \\
Total & 25 & 17 & 42 \\
\hline
\end{tabular}

Abbreviations: $R$ : radiation therapy; $C$ : chemotherapy; $S$ : surgery; $L D$ : limited disease; $E D$ : extensive disease;

*two received adjuvant RT after esophagectomy, and two received pre-operation RT 


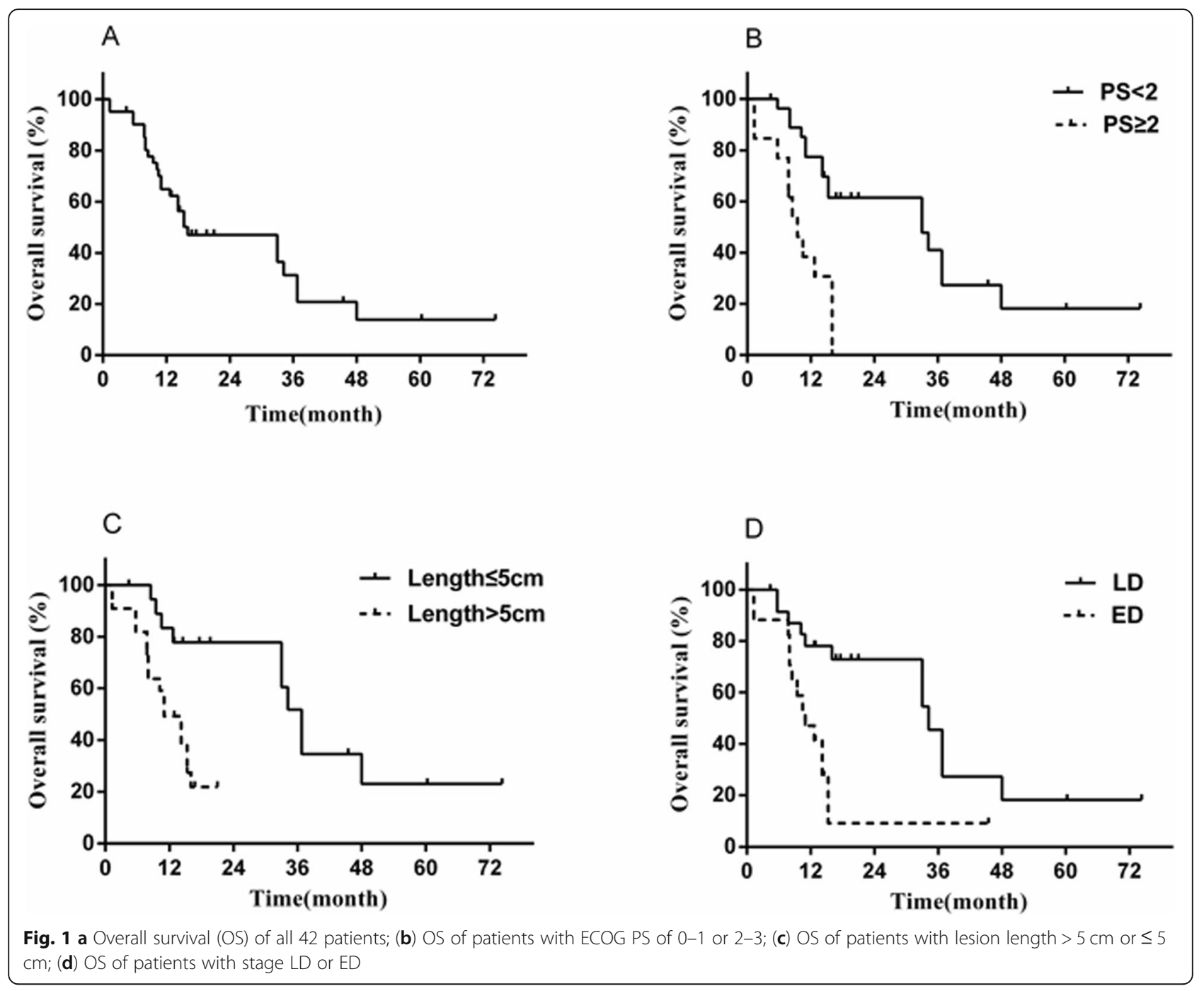

The prognostic factors of the whole cohort and the patients with LD are shown in Table 3. ECOG PS, tumor length, stage, treatment modality (RT vs RT + chemotherapy), RT technology, and radiation dose were included into the Cox multivariate regression model as these variables were significant $(p<0.05)$ in the univariate analysis. Multivariate analysis suggested only ECOG PS, tumor length, and stage are the independent predictors for overall survival in the whole cohort (Fig. 1b-c). Regarding of the patients with LD, multivariate analysis indicated that combined with chemotherapy $(\mathrm{HR}=$ $0.204,95 \%[\mathrm{CI}]=0.050-0.839, P=0.046)$ and a higher radiation dose $(\mathrm{HR}=4.212,95 \%[\mathrm{CI}]=1.024-17.335, P=$ 0.027 ) predicted better prognosis in OS (Fig. 2a-b).

\section{Adverse events}

Most treatment-related toxicities were tolerable and reversible and of grade 1 to 2 . Of the 24 patients who received radiation combined with chemotherapy, grade 3-4 leukocytopenia occurred in five (20.8\%) patients (four with LD and one patient with ED). Other grade 34 toxicity included thrombocytopenia $(n=1)$, esophagitis $(n=1)$, and nausea and vomiting $(n=2)$. The overall rate of grade 3-4 toxicities was $37.5 \%$. Toxicity was lower in 15 patients who received only radiation, of which four (25\%) experienced grade 3-4 toxicity including leukocytopenia $(n=1)$, esophagitis $(n=2)$, and nausea or vomiting $(n=1)$. There was no radiation-induced lung injury or treatment-related death.

\section{Discussion}

Several studies have shown that SCCE only accounts for $0.4-2.8 \%$ of primary esophageal carcinoma $[6,7]$. Reports of increasing incidence of SCCE have come in from East Asian countries, including China and Japan, reaching global trends $[8,9]$. Indeed, a comparison with 
Table 3 Univariate analysis and multivariate Cox regression for prognosis of 42 SCCE patients

\begin{tabular}{|c|c|c|c|c|c|c|}
\hline \multirow[t]{2}{*}{ Factors } & \multicolumn{3}{|c|}{ All cohort $(n=42)$} & \multicolumn{3}{|l|}{$\mathrm{LD}(n=25)$} \\
\hline & $\begin{array}{l}P \text { value } \\
\text { (Univariate) }\end{array}$ & $\begin{array}{l}P \text { value } \\
\text { (Multivariate) }\end{array}$ & $\mathrm{HR}(95 \% \mathrm{Cl})$ & $\begin{array}{l}P \text { value } \\
\text { (Univariate) }\end{array}$ & $\begin{array}{l}P \text { value } \\
\text { (Multivariate) }\end{array}$ & $\mathrm{HR}(95 \% \mathrm{Cl})$ \\
\hline Age ( $<60$ years vs. $\geq 60$ years) & 0.280 & & & 0.989 & & \\
\hline Sex (Male vs. Female) & 0.777 & & & 0.271 & & \\
\hline ECOG PS ( $\geq 2$ vs. $<2$ ) & 0.000 & 0.001 & $6.314(2.027-19.669)$ & 0.048 & 0.291 & $2.577(0.444-14.947)$ \\
\hline Lesion length ( $>5 \mathrm{~cm}$ vs. $\leq 5 \mathrm{~cm}$ ) & 0.001 & 0.001 & $8.593(2.449-30.156)$ & 0.19 & & \\
\hline Smoking history (Yes vs. No) & 0.578 & & & 0.212 & & \\
\hline Risky diet (Yes vs. No) & 0.731 & & & 0.731 & & \\
\hline Stage (ED vs. LD) & 0.002 & 0.049 & $2.786(1.002-7.741)$ & - & - & - \\
\hline Treatment ( R + C vs. R Only) & 0.003 & 0.088 & $0.302(0.076-1.197)$ & 0.028 & 0.046 & $0.204(0.050-0.839)$ \\
\hline Technology (3DCRT vs. IMRT) & 0.039 & 0.941 & $0.948(0.226-3.974)$ & 0.498 & & \\
\hline RT dose (<56Gy vs. $\geq 56 G y)$ & 0.024 & 0.577 & $1.373(0.451-4.184)$ & 0.047 & 0.027 & $4.212(1.024-17.335)$ \\
\hline
\end{tabular}

Abbreviations: $L D$ : limited-disease, ED: extensive-disease, $R T$ : Radiotherapy, $C T$ : Chemotherapy, HR: Hazard Ratio, Cl: Confidence Interval

our previous findings in 2012, the incidence rate of SCCE in our center rose slightly from 0.94 to $1.15 \%$, perhaps due to the improvement in diagnostic pathology [5]. One study described discrepancies in tumor characteristics and therapeutic modalities in different ethnic groups [2]. For instance, the most common primary location of SCCE was the lower thoracic esophagus in the U. S population, while the Chinese population tends to have middle thoracic SCCE. Localized treatment modalities also differ between these two countries. Esophagectomy was the most popularized local therapy in China, which was performed in $\sim 52$ to $85 \%$ of patients $[10,11]$, whereas approximately half of the U.S. patients were reported to receive RT as the principal local therapy, regardless of chemotherapy [2]. Nevertheless, more Asian patients were diagnosed with earlier localized disease and the lack of feasibility of RT during the last few decades might explain this difference. We previously reported that $26.7 \%$ of patients in our center received RT as one part of the treatment, which increased to $40.9 \%$ in the current study, suggesting that RT combined with or without chemotherapy gained more popularity among treating physicians for SCCE recently [5].

The role of RT on the local control of SCCE is not well-illustrated because of the lacking of the information about the response rate in previous reports. In our study, the overall response $(C R+P R)$ rate of $60 \%$ was obtained, suggested that RT alone or combined with chemotherapy was an effective option for locoregional disease control. Also, most published studies focus only on OS, but not PFS or DFS, leading to difficulty in evaluating the efficiency of RT in long-term local control. Our result indicated that the high response rate achieved by RT contributes to the survival benefit through long-duration

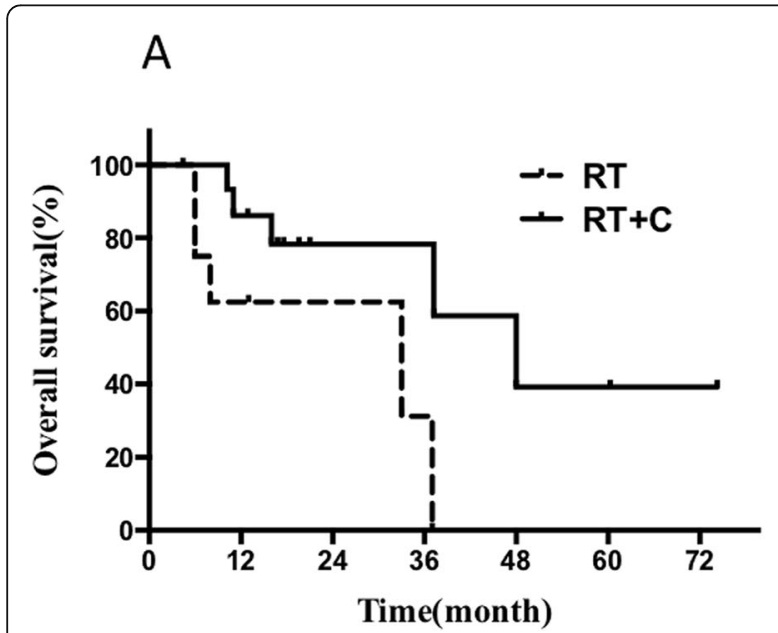

\section{B}

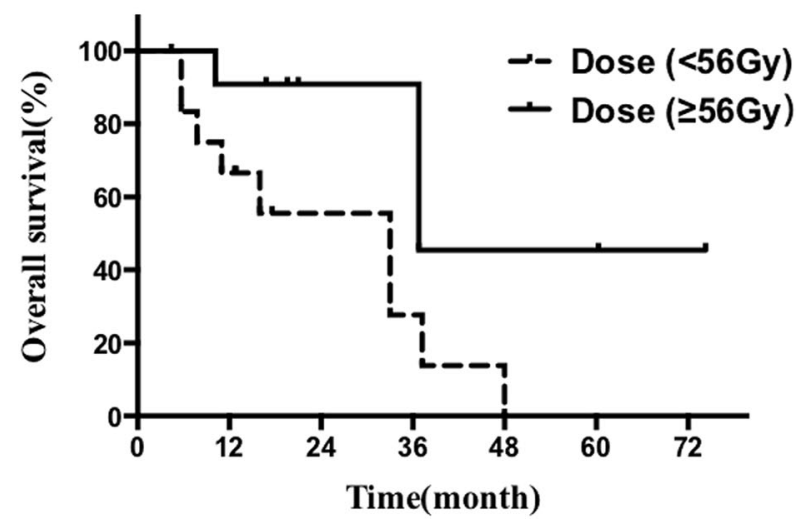

Fig. 2 a OS of the patients with LD stage treated with RT vs RT + C; (b) OS of patients with LD stage treated with lower ( $<56 \mathrm{~Gy})$ vs higher dose( $\geq 56 \mathrm{~Gy})$ of RT 
remission. The median OS was 12.9 months in this study which was similar to previous reports (8-16 months) [2, 4]. The reported OS rates at 1-, 3- and 5-years in SCCE patients who received multiple therapeutics varied from 30 to $74.8 \%, 13.2-38.8 \%$, and $7.8-18 \%$ respectively, and the survival data obtained in our study are almost equivalent to the best outcome [10-13).

As the two main local therapeutic modalities, surgery and definitive RT, especially for LD SCCE, show vast discrepancies when conducted in individuals with different demographics and clinical characteristics. While Sun et al., retrospectively reported 1-, 3- and 5-year OS rates of $50.7,13.7$ and $8.2 \%$ in 73 SCCE patients treated by surgery, these rates are lower than findings we reported previously and here [14]. However, in an analysis of the National Cancer Data Base, esophagectomy was associated with the best OS for patients with localized or local-advanced SCCE comparing to chemoradiation or chemotherapy alone. The 1-, 3- and 5-year OS rates reported in another study were higher than those obtained in our study, but the median overall OS of LD-SCCE patients who received surgery was only 18.0 months, which was consistent with other report but shorter than current analysis $[15,16]$. Interestingly, another study comparing the RT and chemotherapy $(\mathrm{RT}+\mathrm{CT})$ with surgery and chemotherapy $(\mathrm{S}+\mathrm{CT})$ in the management of LD SCCE indirectly favored RT, in which a significantly longer $\mathrm{OS}$ with $\mathrm{RT}+\mathrm{CT}$ compared to $\mathrm{S}+\mathrm{CT}$ (33.0 vs. 17.5 months, $p=0.02$ ) was observed [13]. To sum up, there is a lack of consensus on whether surgery or RT should be the localized treatment modality for SCCE, especially for LD-SCCE, which must be further investigated in prospective controlled studies.

Here we also have shown that RT is also beneficial for the survival of patients with ED SCCE. Similar to the result of the current study, one accumulative analysis of the SEER data demonstrated that the addition of RT leads to a reduction of $30 \%$ risks of death in distant stage ESCC. Despite these promising results, the survival benefit of SCCE from RT was much poorer than that from squamous carcinoma or adenocarcinoma of the esophagus, suggesting the need for novel treatment modalities strategy for SCCE $[17,18]$. Chemotherapy is one of the most important modalities for SCCE, it was wellacknowledged as the fundamental part of the multimodalities therapy and improved the survival both in LD and ED patients $[13,19]$. In our study, the survival of RT + CT group prone to be longer compared to RT alone but without statistically significant difference $(p=0.088)$ of the whole cohort. In patients with $\mathrm{LD}, \mathrm{RT}+\mathrm{CT}$ is correlated with better survival $(p=0.046)$. By summary, the combination of RT and chemotherapy will further improve the survival of SCCE patients. Distant metastasis is the major failure pattern of SCCE despite stages. In accordance with this, over half $(65 \%)$ of patients were failed in the distant control of our study, which further suggested that systemic chemotherapy is essential for the long-term control of SCCE.

The principles of chemotherapy for SCCE is poorly illustrated. According to a study focusing on the SCCE in locoregional disease treated with $\mathrm{RT} \pm$ chemotherapy, more cycles of chemotherapy improved the survival, while the regiment and sequence of RT and chemotherapy had no impact on the disease control [4]. Unfortunately, due to a relatively small size of patients received chemotherapy in our study, we could not yield a reliable analysis to answer these questions. There is no consensus on the practice of RT neither, especially the technology and dose in SCCE, which are based on routine RT for SCLC. The dose used in this study is similar to the common $\mathrm{RT}$ doses ranging from 40-70Gy based on previous case reports and retrospective studies [20-22]. We found that a higher dose of RT ( $\geq 56 \mathrm{~Gy}$ ) is correlated with better survival. However, Jeene et al. reported that no effect of radiotherapy dose on overall survival in the multivariable analysis, while as it was mentioned, the results should have to be interpreted with caution, as only 5 patients received a higher dose of RT in that study. Despite knowing that IMRT is better at target positioning and avoiding errors for esophageal cancer compared to conventional RT [23, 24]. There are no comparative studies of these two RT technologies for SCCE treatment. We did not find significant differences between the OS of patients treated with 3DCRT or IMRT, which might be related to the small sample size of this study.

The main shortcomings of the current study are the small sample size and its retrospective nature. Nevertheless, compared to other studies, we only enrolled the patients who received RT as one part of the treatments and addressed on the local-control role of RT in SCCE by using consistent detailed individual patient data from a single institution. However, the confounding effect caused by enrolling patients with other treatment strategies cannot be overlooked.

Due to its low incidence, prospective and randomized studies on exploring the novel treatments for SCCE is limited. A phase II study (NCT03811379) in our center was initiated in 2018 and is currently recruiting patients to explore the efficacy of toripalimab (PD-1 antibody) as a monotherapy for patients with SCCE, which provides a hint for the novel exploration of a combination of immunotherapy and radiation therapy for SCCE.

\section{Conclusion}

In summary, RT was one of the effective and safe treatment options for locoregional control of SCCE. Lower ECOG PS score, shorter lesion length, treated with chemotherapy, and a higher dose of RT were identified as favorable independent prognostic factors. 


\section{Abbreviations}

3D-CRT: 3-Dimensional Conformal Radiotherapy; CR: Complete remission; CTV: Clinical target volume; ECOG PS: The Eastern Cooperative Oncology Group Performance Status Scale; ED: Extensive disease; GTV: Gross tumor volume; IMRT: Intensity Modulation Radiated Therapy; LD: Limited Disease; OS: Overall survival; PD: Progressive disease; PR: Partial remission; PTV: Planning target volume; RECIST 1.1: Response Evaluation Criteria in Solid Tumors 1.1; RT: Radiation Therapy; SCC: Squamous cell carcinoma; SCCE: Small cell carcinoma of the esophagus; SD: Stable disease

\section{Acknowledgements}

NA

\section{Authors' contributions}

All authors should have made substantial contributions to all of the following: (1) BQC, $H Y, H L M, B Q, Y J Z$, and $Y H H$ contributed to the conception and design of the study. (2) BQC, YH, QQL, and HLM contributed to acquisition of data. (3) BQC, HY, YJZ, and QQL analyzed and interpreted data. (4) BQC and YJZ drafted the article and submitted. All authors revised and approved the final manuscript.

\section{Funding}

This work was supported by Guangdong Esophageal Cancer Institute Science and Technology Program (No.Q201807).

\section{Availability of data and materials}

The datasets used and/or analysed during the current study are available from the corresponding author on reasonable request.

\section{Ethics approval and consent to participate}

This study was approved by the Ethics Committee of Sun Yat-sen University Cancer Center. Written informed con- sent was obtained from the patient for the publication of this report.

\section{Consent for publication}

NA.

\section{Competing interests}

The authors declare that they have no competing financial interests.

\section{Author details}

'Department of Radiation Oncology, State Key Laboratory of Oncology in South China, Collaborative Innovation Center of Cancer Medicine, Sun Yat-sen University Cancer Center, Guangzhou, 510060, P. R. China. ${ }^{2}$ Guangdong Esophageal Cancer Institute, Guangzhou, P. R. China. ${ }^{3}$ Department of Thoracic Surgery, State Key Laboratory of Oncology in South China, Collaborative Innovation Center of Cancer Medicine, Sun Yat-sen University Cancer Center, Guangzhou, 510060, P. R. China. ${ }^{4}$ Department of Radiology, State Key Laboratory of Oncology in South China, Collaborative Innovation Center of Cancer Medicine, Sun Yat-sen University Cancer Center, Guangzhou, 510060, P. R. China.

Received: 12 July 2019 Accepted: 4 November 2019

\section{Published online: 21 November 2019}

\section{References}

1. McKeown F. Oat-cell carcinoma of the oesophagus. J Pathol Bacteriol. 1952; 64(4):889-91.

2. Xiao Q, Xiao H, Ouyang S, Tang J, Zhang B, Wang H. Primary small cell carcinoma of the esophagus: comparison between a Chinese cohort and surveillance, epidemiology, and end results (SEER) data. Cancer Med. 2019; 8(3):1074-85

3. Wong AT, Shao M, Rineer J, Osborn V, Schwartz D, Schreiber D. Treatment and survival outcomes of small cell carcinoma of the esophagus: an analysis of the National Cancer Data Base. Dis Esophagus. 2017;30(2):1-5.

4. Jeene PM, Geijsen ED, Muijs CT, Rozema T, Aleman BMP, Muller K, et al. Small cell carcinoma of the esophagus: a Nationwide analysis of treatment and outcome at patient level in Locoregional disease. Am J Clin Oncol. 2019;42(6):534-8.
5. Zhu Y, Qiu B, Liu H, Li Q, Xiao W, Hu Y, et al. Primary small cell carcinoma of the esophagus: review of 64 cases from a single institution. Dis Esophagus. 2014;27(2):152-8.

6. Sasajima K, Watanabe M, Ando T, Hao K, Miyashita M, Yamashita K, et al. Serum neuron-specific enolase as a marker of small-cell carcinoma of the esophagus. J Clin Gastroenterol. 1990;12(4):384-8.

7. Krishnatreya M, Kataki AC, Sharma JD, Borthakur BB, Kalita M. Epidemiology of primary small cell carcinoma of the esophagus: a retrospective study. South Asian J Cancer. 2014;3(4):231-2.

8. Tao H, Li F, Wang J, Dong W, Gao J, Jiao S, et al. Management of treatmentnaive limited-stage small cell esophagus carcinoma. Saudi Med J. 2015;36(3): 297-303.

9. Nayal B, Vasudevan G, Rao AC, Kudva R, Valliathan M, Mathew M, et al. Primary small cell carcinoma of the esophagus - an eight year retrospective study. J Clin Diagn Res. 2015:9(5):EC04-6.

10. Xie MR, Xu SB, Sun XH, Ke L, Mei XY, Liu CQ, et al. Role of surgery in the management and prognosis of limited-stage small cell carcinoma of the esophagus. Dis Esophagus. 2015;28(5):476-82.

11. Lu XJ, Luo JD, Ling Y, Kong YZ, Feng LL, Zhou J, et al. Management of small cell carcinoma of esophagus in China. J Gastrointest Surg. 2013;17(7):1181-7.

12. Lu JM, Liang J, Wang JW, He J, Xiao ZF, Zhang HX, et al. Clinical analysis of 126 patients with primary small cell carcinoma of the esophagus. Zhonghua Zhong Liu Za Zhi. 2009;31(2):121-5

13. Meng MB, Zaorsky NG, Jiang C, Tian LJ, Wang HH, Liu CL, et al. Radiotherapy and chemotherapy are associated with improved outcomes over surgery and chemotherapy in the management of limited-stage small cell esophageal carcinoma. Radiother Oncol. 2013;106(3):317-22.

14. Sun KL, He J, Cheng GY, Chai LX. Management of primary small cell carcinoma of the esophagus. Chin Med J. 2007;120(5):355-8.

15. Situ $D$, Lin $Y$, Long $H$, Zhang $L$, Lin $P$, Zheng $Y$, et al. Surgical treatment for limited-stage primary small cell cancer of the esophagus. Ann Thorac Surg. 2013;95(3):1057-62.

16. Ding J, Ji J, Zhu W, Zhou K, Han J, Zhang Y, et al. A retrospective study of different treatments of limited-stage small-cell esophageal carcinoma and associated prognostic factor analysis. Dis Esophagus. 2013;26(7):696-702.

17. Ohashi S, Miyamoto S, Kikuchi O, Goto T, Amanuma Y, Muto M. Recent advances from basic and clinical studies of esophageal squamous cell carcinoma. Gastroenterology. 2015;149(7):1700-15.

18. Kim E, Koroukian S, Thomas CR Jr. Conditional survival of esophageal Cancer: an analysis from the SEER registry (1988-2011). J Thorac Oncol. 2015 10(10):1490-7.

19. Zhang WN, Chen YP, Chen L, Guo R, Zhou GQ, Tang LL, et al. The Clinical Utility of Plasma Epstein-Barr Virus DNA Assays in Nasopharyngeal Carcinoma: The Dawn of a New Era? Medicine (Baltimore). 2015:94(20).

20. Atsumi K, Shioyama Y, Nomoto S, Ohga S, Toba T, Sasaki T, et al. Chemoradiation for small cell esophageal carcinoma: report of 11 cases from multi-institution experience. J Radiat Res. 2010;51(1):15-20.

21. Nevarez A, Saftoiu A, Bhutani MS. Primary small cell carcinoma of the esophagus: Clinico- pathological features and therapeutic options. Curr Health Sci J. 2011:37(1):31-4.

22. Chen SB, Yang JS, Yang WP, Weng HR, Li H, Liu DT, et al. Treatment and prognosis of limited disease primary small cell carcinoma of esophagus. Dis Esophagus. 2011;24(2):114-9.

23. Han C, Schiffner DC, Schultheiss TE, Chen YJ, Liu A, Wong JY. Residual setup errors and dose variations with less-than-daily image guided patient setup in external beam radiotherapy for esophageal cancer. Radiother Oncol. 2012:102(2):309-14.

24. Hawkins MA, Aitken A, Hansen VN, McNair HA, Tait DM. Set-up errors in radiotherapy for oesophageal cancers--is electronic portal imaging or conebeam more accurate? Radiother Oncol. 2011;98(2):249-54.

\section{Publisher's Note}

Springer Nature remains neutral with regard to jurisdictional claims in published maps and institutional affiliations. 